\title{
Energy Efficiency Evaluation of EV Charging Network Based on Fuzzy Comprehensive Assessment
}

\author{
Yubo Fan, Chunlin Guo, Wen Chen, Zongfeng Li \\ State Key Laboratory of Alternate Electrical Power System with Renewable Energy Sources \\ North China Electric Power University \\ Beijing ,China \\ jianhufanyubo@163.com
}

\begin{abstract}
People began to focus on the energy efficiency of EV charging facilities with the rapid development of $\mathrm{EV}$. The energy efficiency evaluation of EV charging network requires a comprehensive reflection of the entire and all aspects of the network. This paper aims to propose a method and a model of energy efficiency evaluation. Firstly EV efficiency factors were analyzed, which were conducted hierarchical classification, and then to calculate factor weights using the G1 group method. Finally energy efficiency evaluation model was established by the analysis of factor set, evaluation set, weighting factor based on fuzzy comprehensive evaluation principle. Simulation results show that the result of energy efficiency evaluation by this model not only contained the retail network energy efficiency, but also reflected the various aspects of the network. The model reflected energy efficiency of the whole network meticulously and roundly, which has a strong practical value.
\end{abstract}

Keywords-component; electric vehicle; energy efficiency; fuzzy comprehensive assessment; G1 group

\section{INTRODUCTION}

As a type of New Energy Vehicles, EV possess the advantage that fuel vehicles are beyond comparison and come into being the research emphasis in the world. In China, the development of the new energy automotive industry, dominated by EV, has listed in "the 12th Five-Year Plan", becoming a major project in promoting energy conservation, emission reduction and updating the industry ${ }^{[1-2]}$.

When EV is in large-scale application, the research on energy efficiency of charging facilities, serving as a type of conversion of electrical energy device ,along with the establishment of integrated EV charging evaluation system, contribute to enhancing the operation efficiency of charging facilities, and guarantee the optimization in equipment use and system operation, which have great social value ${ }^{[3-4]}$.

Therefore, for the energy efficiency evaluation of EV charging network, we conducted comprehensive hierarchical evaluation by using fuzzy set theory in this paper. Dividing $\mathrm{EV}$ efficiency factors into two grades and conducting comprehensive hierarchical evaluation by fuzzy comprehensive evaluation method, help to understand the energy efficiency level of the whole network, as well as the energy efficiency level of the each charging facilities in

*This work is supported by: National High Technology R\&D Program of China (863Program) (2012AA050804). different operation mode and charging mode ${ }^{[5-6]}$.

In order to study the efficiency factors of EV charging network, this paper ignored internal circuit loss in power electronic devices such as motors, and study the electric conversion and utilization efficiency form the whole behavior of charging electric.

According to the researches in REF[7-8],there can be 6 factors that may have influence on the EV charging network which will be discussed in the following part.

\section{THE ENERGY EFFICIENCY RESEARCH BASED ON THE FUZZY COMPREHENSIVE EVALUATION METHOD}

The fuzzy comprehensive evaluation method is an effective, multi-factors decision making method that can evaluate many issues which can be influenced by many factors in various aspects ${ }^{[9]}$.

When we use the fuzzy comprehensive assessment, let the $U=\left\{u_{1}, u_{2}, \cdots u_{n}\right\}$ be the $\mathrm{n}$ factors and $V=\left\{v_{1}, v_{2}, \cdots v_{m}\right\}$ be the $m$ evaluations. However, these factors lies in different aspects, thus the weight of factors can be different. And comprehensive evaluation should be V on a fuzzy subset.

$$
\underset{\mathrm{O} \%}{B}=\left(b_{1}, b_{2}, \cdots, b_{m}\right) \in F(v)
$$

In the formula above, the entire fuzzy subsets of the universe of $\mathrm{V}$ consist $F(v): b_{j}(j=1,2, \cdots, m)$ is the subordinate degree of $v_{j}$ to fuzzy set $\underset{0 /}{B}, \underset{0}{B}\left(v_{j}\right)=b_{j}$; the integrated evaluation set $B$ depends on the subordinate degree of each factor, which means the subordinate degree of each factor is a fuzzy subset of $\mathrm{U}: A=\left(a_{1}, a_{2}, \cdots, a_{n}\right) \in F(U), \sum_{i=1}^{n} a_{i}=1$, where $a_{i}$ denotes the weight of $\mathrm{i}$-th factors. If given the weight set $\mathrm{A}$, we can calculate a integrated evaluation set $B$.

Considering the ambiguity of the EV charging network, we classified the factors that is related to EV charging network efficiency to get a hierarchical clustering ${ }^{[13]}$. Firstly we use fuzzy comprehensive evaluation method to assess the lower index, then the senior index. Figure 1 shows the procedures of Fuzzy Comprehensive Assessment Method evaluation. 


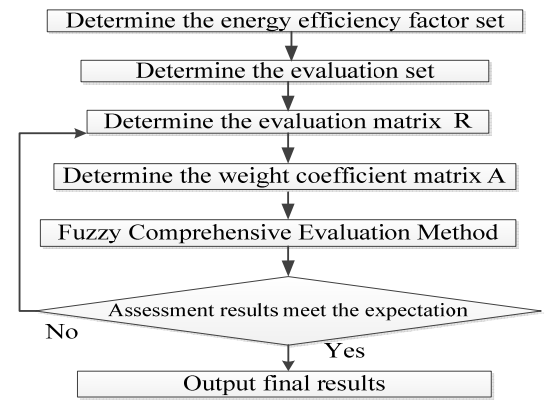

Figure 1. Procedure of fuzzy comprehensive assessment

\section{A. Determine the energy efficiency factor set Abbreviations and Acronyms}

Combining the actual live investigation and the efficiency research of factors of EV charging facilities, a system for evaluating EV energy efficiency was established $^{[10-11]}$. Then we made three charging voltages: ${ }_{U}$ for dispersion slow charging; $U_{2}$ for fast charging; $U_{3}$ for charging stations. As for dispersion low charging station and fast charging station, we established secondary energy efficiency indicator of EV charging station as followings: the efficiency of distribution network, the efficiency of transformers, the efficiency of charger, the efficiency of power transfer facilities, the energy efficiency of power plant position and the energy efficiency of battery distribution. The assessment model overall were shown in Figure 2, the energy efficiency set is as follows ${ }^{[9]}$.

$U=\left\{U_{1}, U_{2}, U_{3}\right\} \quad$, where $U_{1}=\left\{u_{11}, u_{12}, u_{13}\right\} \quad U_{3}=\left\{u_{21}, u_{22}, u_{23}\right\}$

$U_{3}=\left\{u_{31}, u_{32}, u_{33}, u_{34}, u_{35}, u_{36}\right\}$

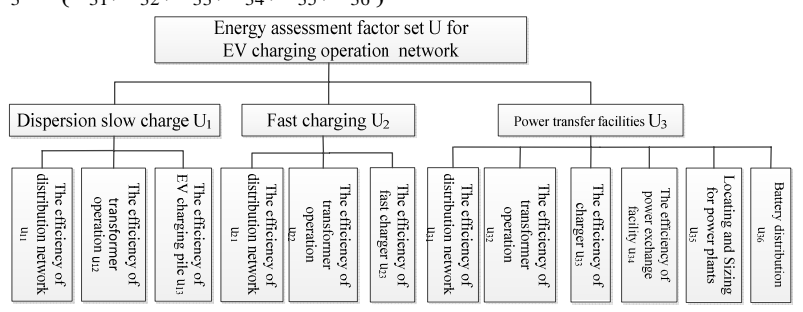

Figure 2. Evaluation model of EV charging network

\section{B. Determine the evaluation set}

Let $V=\left\{v_{1}, v_{2}, v_{3}, \cdots, v_{n}\right\}$ be the assessment set of efficiency for EV charging network. And the set is suitable for all assess factors. However, the establishment of $\mathrm{V}$ has a vital impact for the efficient evaluation for EV charging. According to issue we discussed in part 1, we divided the $\mathrm{EV}$ energy efficiency evaluation stage into six indicators which are shown in TABLE I.
TABLE I. ENERGY EFFICIENCY EVALUATION SET OF EV CHARGING NETWORK

\begin{tabular}{|c|c|}
\hline $\begin{array}{c}\text { Indicators } \\
\text { level }\end{array}$ & content \\
\hline $\begin{array}{c}\text { Fist grade } V_{1} \text { 错 } \\
\text { 误! 未找到引 } \\
\text { 用源。 }\end{array}$ & Lowest energy efficiency, the most unreasonable use of \\
power \\
\hline $\begin{array}{c}\text { Second grade } \\
V_{2}\end{array}$ & Lower energy efficiency. more unreasonable use of power \\
\hline Third grade $V_{3}$ & Low energy efficiency, unreasonable use of power \\
\hline Fourth grade $V_{4}$ & Average energy efficiency, reasonable use of power \\
\hline Fifth grade $V_{5}$ & High energy efficiency, more reasonable use of power \\
\hline Sixth class $V_{6}$ & Highest energy efficiency, the most reasonable use of power \\
\hline
\end{tabular}

C. Determine the assessment matrix basing on the fuzzy comprehensive evaluation method

For single factor assessment, by the fuzzy mathematics formula :

$$
\begin{aligned}
\underset{\sim}{f}: U & \rightarrow F(V) \\
u_{i} \mapsto \underset{\sim}{f}\left(u_{i}\right)=\left(r_{i 1}, r_{i 2}, \cdots,\right. & \left.r_{i m}\right) \in F(V)
\end{aligned}
$$

The fuzzy map $f$ could gain the fuzzy-relation set $\underset{\sim}{R} \in F(U \times V)$, which means $R_{f}\left(u_{i}, v_{i}\right)=f\left(u_{i}\right)\left(v_{i}\right)=r_{i j}$. Thus we can show $\underset{\sim}{R_{f}}$ with a fuzzy matrix named $R \in u_{n \times m}:$

$$
R=\left[\begin{array}{cccc}
r_{11} & r_{12} & \cdots & r_{1 m} \\
r_{21} & r_{22} & \cdots & r_{2 m} \\
\vdots & \vdots & & \vdots \\
r_{n 1} & r_{n 2} & \cdots & r_{n m}
\end{array}\right]
$$

Where $r_{i j}$ is the subordinate degree of the i-th energy efficiency factor set to the $\mathrm{j}$-th assessment set, which is decided by the subordinate degree function:

$$
r_{i j}=f_{i j}=\sum_{j=1}^{n} f_{i j}
$$

The $f_{i j}$ in the formula is the power of the i-th energy efficiency factor $u_{i}$ set to the $\mathrm{j}$-th assessment set $v_{j}$.

\section{Determine the weight coefficient matrix}

The weight of indicators is somehow reflection of the related importance in the assessment system. However, it is always the key issue that how to determine the weight coefficient ${ }^{[12]}$.

The process of G1 method which is also called the rank correlation analysis is as follows:

First: determine the rank relation

In the process of G1 method, if the importance of the relative evaluation criteria of some certain energy efficiency factor $u_{i}$ is greater than that of $u_{j}$, we mark $u_{i}>u_{j}$. And if the evaluation factors $u_{1}, u_{2}, u_{3} \cdots, u_{n}$ has the relation as follows : $u_{1}>u_{2}>u_{3} \cdots>u_{m}$, then we call the assessment factors are orderly by " $>$ ”. 
Second: determine the value of $S_{k}$

After determining the ordering relation, we used $S_{k}$ to demonstrate the ratio of importance of $u_{k-1}$ and $u_{k}$ in the energy efficiency set U:

$$
S_{k}=a_{k-1} / a_{k}
$$

Where $a_{k}$ represent the weight of the k-th factor in the energy efficiency set $U$. The meaning of $s_{k}$ was shown in the TABLE II.

TABLE II. ORDER RELATION AND IMPORTANCE INDEX

\begin{tabular}{|c|c|}
\hline$S_{K}$ & Description \\
\hline 1.0 & $u_{k}$ has the same importance as $u_{k-1}$ \\
\hline 1.2 & $u_{k-1}$ is a little more important than $u_{k}$ \\
\hline 1.4 & $u_{k-1}$ is more important than $u_{k}$ obviously \\
\hline 1.6 & $u_{k-1}$ is more important than $u_{k}$ strongly \\
\hline 1.8 & $u_{k-1}$ is more important than $u_{k}$ extremely \\
\hline
\end{tabular}

Third: calculating the weight coefficient of $a_{k}$

$$
\begin{aligned}
& a_{n}=\left(1+\sum_{k=2}^{n} \prod_{i=k}^{n} s_{i}\right)^{-1} \\
& a_{k-1}=s_{k} a_{k}(k=n, n-1, \ldots, 3,2)
\end{aligned}
$$

Fourth step: determine the comprehensive weight

When we hire $L$ experts to evaluate a same issue, due to the different ordering relations given by different experts ,there may be two kinds of possibilities that: the $L$ ordering relation are consistent or the $L$ ordering relations are inconsistent.

Let the $L_{1}$ experts $\left(1 \leqslant L_{1} \leqslant L\right.$ ) of the $L$ experts gave the same ordering relation, and we can use $S_{i h}$ to show the value of $s_{i}$, where $h=1,2, \cdots L_{1}$ :

$$
S_{i}=\frac{1}{L_{1}} \sum_{h=1}^{L 1} s_{i h}
$$

Put the formula (9) in the formula (8) and (7),and we can calculate the weight coefficient $a_{i}^{(1)}$ of the $L_{1}$ experts determined. After that, we continue to seek if there were some same ordering relations in the $L-L_{1}$ experts, and if there existed a same ordering relations, we will calculate the weight $a_{i}^{(2)}, \cdots$ according to the formula (7) to (9) until the rest of the $L_{e}$ ordering relation are all different. Thus we calculated the every weight coefficient made by the $L_{e}$ experts, and then calculate the arithmetic average comprehensive value $p_{i}^{(e)}$. At last, the weight coefficient determined by $L$ experts are as follows:

$$
A=c_{1} p_{i}^{(1)}+c_{2} p_{i}{ }^{(2)}+\ldots c_{e} p_{i}{ }^{(e)}
$$

Where $c_{i}=L_{i} / L$ is the proportion of a certain weight coefficient determined by a certain expert to all experts.

\section{E. Determine the model of the comprehensive evaluation}

We used the hierarchical comprehensive assessment method to evaluate the energy efficiency of EV charging network, and gained the comprehensive evaluation results of factor set $U_{i}$ by complex arithmetic:

$$
\begin{gathered}
B_{i}=A_{i} \circ R_{i}=\left(b_{i 1}, b_{i 2}, \cdots, \quad b_{i m}\right) \\
b_{i j}=\sum_{k=1}^{n}\left(a_{i k} r_{i k}\right) \quad 1 \leq j \leq m
\end{gathered}
$$

After we gained the evaluation results of the second grade factor set, we evaluated the first grade factor set which meant that we evaluated the fuzzy assessment space $(U, V, R)$. However, the assessment matrix $R$ of the factor set $U$ was consisted of the comprehensive output $B_{i}$ of the second factor :

$$
R=\left[\begin{array}{c}
B_{1} \\
B_{2} \\
\vdots \\
B_{L}
\end{array}\right]=\left[\begin{array}{ccc}
A_{1} & \circ & R_{1} \\
A_{2} & \circ & R_{2} \\
\vdots & \vdots & \vdots \\
A_{L} & \circ & R_{L}
\end{array}\right]
$$

Above all, the final comprehensive evaluation system of the energy efficient evaluation factor set $u$ of the EV charging network is shown as follows:

$$
B=A \circ R=\left(b_{1}, b_{2}, \cdots, b_{m}\right)
$$

\section{EXAMPLES ANALYSIS OF ENERGY EFFICIENCY EVALUATION}

Select a certain region in some typical city, where there are several dispersed charging piles, rapid charging station and EV charging station. The energy efficiency evaluation system is showed in Fig.[2].

\section{A. Calculation of energy efficiency evaluation}

Adopting the G1 group method introduced above to calculate each factor weight, we get the energy efficiency factor set weights

$$
A=\left(\begin{array}{lll}
0.3 & 0.4 & 0.4
\end{array}\right)
$$

Second-level factor set weights are

$A_{3}=\left(\begin{array}{lll}0.2 & 0.2 & 0.6\end{array}\right)$

$A_{2}=\left(\begin{array}{lll}0.2 & 0.3 & 0.5\end{array}\right)$

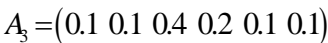

By fuzzy comprehensive evaluate U1,we get fuzzy evaluation matrix

$$
R_{1}=\left[\begin{array}{cccccc}
0 & 0 & 0.1 & 0.2 & 0.5 & 0.2 \\
0 & 0 & 0.1 & 0.5 & 0.4 & 0 \\
0 & 0.1 & 0.2 & 0.6 & 0.1 & 0
\end{array}\right]
$$

The evaluation result of $U_{1}$ :

$$
B_{1}=A_{1} \circ R_{1}=\left[\begin{array}{l}
0.2 \\
0.2 \\
0.6
\end{array}\right]^{\mathrm{T}}\left[\begin{array}{lllllc}
0 & 0 & 0.1 & 0.2 & 0.5 & 0.2 \\
0 & 0 & 0.1 & 0.5 & 0.4 & 0 \\
0 & 0.1 & 0.2 & 0.6 & 0.1 & 0
\end{array}\right]=
$$$$
=\left[\begin{array}{llllll}
0 & 0.06 & 0.16 & 0.5 & 0.24 & 0.04
\end{array}\right]
$$

By the sane way, we get The evaluation result of $U_{2}$, $U_{3}, U_{4}$

$$
B_{2}=A_{2} \circ R_{2}=\left[\begin{array}{llllll}
0 & 0.03 & 0.08 & 0.63 & 0.24 & 0.02
\end{array}\right]
$$


$B_{3}=A_{3} \circ R_{3}=\left[\begin{array}{llllll}0.02 & 0.06 & 0.19 & 0.54 & 0.13 & 0.06\end{array}\right]$

Therefore we get fuzzy evaluation matrix of energy efficiency set $\mathrm{U}$ :

$$
R=\left[\begin{array}{l}
B_{1} \\
B_{2} \\
B_{3}
\end{array}\right]=\left[\begin{array}{cccccc}
0 & 0.06 & 0.16 & 0.5 & 0.24 & 0.04 \\
0 & 0.03 & 0.08 & 0.63 & 0.24 & 0.02 \\
0.02 & 0.06 & 0.19 & 0.54 & 0.13 & 0.06
\end{array}\right]
$$

Then we get the result of energy efficiency evaluation of EV charging network after criticism:

$$
B=A \circ R=\left[\begin{array}{llllll}
0.008 & 0.051 & 0.148 & 0.555 & 0.196 & 0.042
\end{array}\right]
$$

\section{B. Result analysis}

By calculating, the result of energy efficiency evaluation of EV based on fuzzy comprehensive strategy is showed in Tab.[3]

TABLE III. RESULTS OF ENERGY EFFICIENCY ASSESSMENT

\begin{tabular}{|c|c|c|c|c|c|c|}
\hline \multirow{2}{*}{$\begin{array}{c}\text { The factor } \\
\text { of energy } \\
\text { efficiency }\end{array}$} & \multicolumn{7}{|c|}{$\begin{array}{c}\text { Evaluation results } \\
\text { one }\end{array}$} & $\begin{array}{c}\text { Grade } \\
\text { two }\end{array}$ & $\begin{array}{c}\text { Grade } \\
\text { three }\end{array}$ & $\begin{array}{c}\text { Grade } \\
\text { four }\end{array}$ & $\begin{array}{c}\text { Grade } \\
\text { five }\end{array}$ & $\begin{array}{c}\text { Grade } \\
\text { six }\end{array}$ \\
\hline$U$ & 0.08 & 0.051 & 0.148 & 0.555 & 0.196 & 0.042 \\
\hline$U_{1}$ & 0 & 0.06 & 0.16 & 0.5 & 0.24 & 0.04 \\
\hline$U_{2}$ & 0 & 0.03 & 0.08 & 0.63 & 0.24 & 0.02 \\
\hline$U_{3}$ & 0.02 & 0.06 & 0.19 & 0.54 & 0.13 & 0.06 \\
\hline
\end{tabular}

Among the result of energy efficiency evaluation of EV charging network, there 6 memberships graded 0.555 are maximum, Based on maximum membership principle, the evaluation result is graded the fourth-stage: energy efficiency is general and energy consumption is reasonable. This result indicated that energy efficiency is general and energy consumption is reasonable in generally.

In the evaluation set, three grades including the fourth, the fifth and the sixth grade are treated as the case that the level of energy efficiency and energy consumption are reasonable. We could regard the factors rated as the fourth, the fifth or the sixth stage as reaching the standard. In the Fig about the comprehensive evaluation of EV charging network, about $89.3 \%$ evaluation experts agree the integral energy efficiency level of EV in this region reaches the standard. As for each charging link, about 78\% evaluation experts agree dispersed slow charging stations reach the standard. About 89\% evaluation experts agree rapid charging stations reach the standard, while about $73 \%$ evaluation experts agree charging stations reach the standard

For further analysis, we might as well rank the energy efficiency evaluation set by score, where the total points is 100.Grade 0,30,50,60,80 and 100 from the first-stage to the sixth-stage, respectively and get the point table as TABLE IV

TABLE IV. RESULTS OF ENERGY EFFICIENCY ASSESSMENT

\begin{tabular}{|c|c|c|}
\hline Factor of energy efficiency & Point of evaluation & Rank \\
\hline$U_{1}$ & 63 & 2 \\
\hline$U_{2}$ & 63.9 & 1 \\
\hline$U_{3}$ & 60.1 & 3 \\
\hline
\end{tabular}

We can know from the Fig that the all factors of each charging link reach the standard, while the energy efficiency level is near qualified state and has a huge rising space.

\section{CONCLUSION}

This paper proposed a method and a model of energy efficiency evaluation by studying the EV charging network efficiency factor. Given the fuzziness of energy efficiency evaluation, the EV efficiency factors were conducted hierarchical evaluation by using fuzzy comprehensive evaluation method in this paper. This method not only contained the various aspects of the network, but also reflected the retail network energy efficiency. With the example verification, this method proved a great practical value.

\section{REFERENCES}

[1] Zhang Wenliang, Wu Bin,Li, Wufeng, etal .Discussion on development trend on battery electric vehicles in China and its energy supply mode[J]. Power System Technology, 2009, 33(4) : 15(in Chinese).

[2] Yang Xiaolun. The development trend and foreground of the electric vehicle[J]. Auto Mobile Science \& Technology, 2007(6):10-13(in Chinese). .

[3] Energy Information Administration, US Department of Energy. Annual energy review[R]. Washington DC:US Department of Energy, 2010

[4] John V. Top 10 tech cars[R]. USA : The Institute of Electrical and Electronics Engineers,2007

[5] 2010[2011-07-

01],http://money.163.com/10/0712/04/6BC8HRIN002534NV.html.

[6] Du Jian,Xie Dong, Wu Tao, et al. Status of foreign electric vehicle development strategy and charge-exchange service[J]. Central China Electric Power, 2012, 25(1) : 96-102(in Chinese).

[7] LI Huiling,BAI xiaomin. Impacts of Electirc Vehicles Charging on Distribution Grid[J].Automation of Electric Power Systems,2011(17):38-42(in Chinese).

[8] Kuss M,Markel T,Kramer W. Application of distribution transformer thermal life models to electrified vehicle charging loads using Monte-Carlo method[C]//The 25th World Battery, Hybrid and Fuel Cell Electric Vehicle Symposium \& Exhibition. Shenzhen, China : Electric Vehicle Association of Asia Pacific, 2010:1-8.

[9] Xie Ji-jian, Liu Cheng-ping ,Fuzzy mathematics and the application[M]. Wu Han,Huazhong University of Science and Technology Press,2013:2

[10] LIAO Rui-jin, WANG Qian, et al. Condition assessment model for power transformer in service based on fuzzy synthetic evaluation[J]. Automation of Electric Power Systems, 2008, 32(3):70-74

[11] ZHOU Li-sha , YU Xin-hua. Fuzzy comprehensive evaluation of power customer satisfaction bassed on analytic network process[J]. Power System Technology, 2009, 33(17):191-197

[12] ZHENG Xue-ru, HE Jian-min. The method of define the weight of DSM's factor bassed on AHP[J]. Modern Management Science, 2006(8):8-9 